\title{
REPORTES FINANCIEROS Y LA TOMA DE DECISIONES EN LAS
}

\author{
ORGANIZACIONES
}

\section{FINANCIAL REPORTING AND DECISION MAKING IN}

\author{
ORGANIZATIONS
}

\author{
José Véliz ${ }^{1}$, Raúl Carpio ${ }^{2}$
}

\section{Palabras clave: \\ Reportes \\ Financieros, \\ Toma de \\ Decisiones, \\ Generación de \\ Valor}

\begin{abstract}
Resumen
Los Reportes Financieros constituyen indicadores del bienestar de las organizaciones, por eso, históricamente han sido considerados en la Toma de Decisiones, manteniendo una relación de dependencia. Con base a este antecedente, el presente estudio tiene como finalidad estudiar dicha relación, mediante una minuciosa revisión de la literatura de los principales conceptos referentes a la toma de decisiones y los estados financieros de los estudios realizados durante los últimos diez años. Así mismo, esta investigación explaya el perfil de los tomadores de decisiones y cómo esto mejora el impacto de tales decisiones. Se concluye que la información financiera genera múltiples beneficios, entre ellos: diversificación de la participación en las decisiones, incremento de la generación de valor y mayor credibilidad de la entidad frente a inversionistas y otros actores.
\end{abstract}

Códigos JEL: M10, M40.

\footnotetext{
Keywords:

Financial reports, Decision making, Value Generation

Abstract

Financial Reports are indicators of the well-being of organizations, therefore, historically they have been considered in Decision Making, maintaining a relationship of dependency. Based in this background, the purpose of this study is to study this relationship by means of a meticulous revision of the literature of the main concepts

${ }^{1}$ Pontificia Universidad Católica del Perú, Graduate Business School, CETRUM Católica, Lima, (Perú). E-mail: jcveliz@pucp.edu.pe 
referring to decision-making and financial statements, during the last ten years. Furthermore, this research explains the profile of decision makers and how this improves the impact of such decisions. It is concluded that financial information generates multiple benefits, such as diversification of participation in decision making, greater generation of value and credibility of the company to investors and other actors.

JEL Codes: M10, M40.

\section{INTRODUCCIÓN}

En la actualidad, las organizaciones definen un modelo de negocio que representa la declaración base de la empresa, de la cual se espera generar ingresos y beneficios. Dicho modelo establece los atributos de valor específicos que una empresa ofrece a sus clientes en un producto o servicio y que la diferencian de la competencia. Esto significa que las operaciones de la empresa y todas las actividades incluyendo la mayoría de las decisiones de la gerencia, deben estar alineadas con su propuesta de valor (Collins, Román, \& Chan, 2011).

Además, la propuesta de valor debe estar alineada a la estrategia y reflejarse en indicadores financieros, de manera que se pueda determinar si las actividades se realizan de manera correcta y oportuna. Según Bernard, Dupuy, Baker \& Blum (2018), esto tiene un notable impacto en los beneficios futuros y el incremento del valor patrimonial de la organización.

En ese sentido, Cheng, Young \& Yang (2018), indican que los informes financieros mejoran las decisiones de inversión y asignación de capital de las empresas. En su investigación proporcionan dos argumentos no exclusivos sobre cómo los cambios en la información financiera afectan las decisiones corporativas de las empresas. La primera se basa en los costos de los organismos: la mejora de los informes financieros externos puede reducir la asimetría de información entre los administradores y los accionistas y, por ende, los costos de los organismos, lo que conduce a mejores decisiones institucionales. El segundo argumento se basa en el cambio en el entorno interno de información debido a los cambios en los informes externos, los gerentes deben recopilar más información para cumplir con los nuevos estándares. A través de este proceso, es probable que los gerentes obtengan nueva información y esta les ayude a tomar mejores decisiones corporativas.

Dada la relación existente entre los estados financieros y la toma de decisiones para generar una propuesta de valor, el presente trabajo tiene como finalidad estudiar dicha relación mediante una minuciosa revisión de la literatura de los principales conceptos relacionados a la toma de decisiones y los estados financieros, durante los últimos diez años.

\section{METODOLOGÍA}

La metodología de este estudio consiste en la revisión de literatura, para lo cual se utilizó la base de datos SCOPUS. Cabe mencionar que, durante la búsqueda, se denotó que en Estados Unidos se realiza la mayor producción intelectual referente al tema de este trabajo. Por tanto, se empleó mayormente fuentes provenientes de ese país.

Luego, se procedió a discutir y analizar los aportes de cada fuente consultada, considerando de forma esencial todas aquellas que se alineaban más al tema en cuestión. Esto resultó en una lista de fuentes principales, la cual se decidió exponer a modo de tabla resumen (ver apéndice), de manera que pueda servir de guía para futuras investigaciones.

\section{RESULTADOS}

En lo que respecta a reportes financieros, estos tienden a ser una fuente para la toma de decisiones 
en las empresas y, generalmente, los gerentes de finanzas (CFO) son los responsables de la presentación de los informes financieros y de la transparencia de la información comunicada; ya que esta proporciona el retrato más completo y realista posible de los impactos positivos y negativos de las actividades empresariales en general (Bernard, Dupuy, Baker, \& Blum, 2018).

Al respecto, Boiral (2013) comenta que la contabilidad de los instrumentos financieros parece haberse convertido en aquella en que los resultados contables se desprenden de la realidad económica subyacente y esto impacta en los resultados financieros de la organización y, por consiguiente, en las decisiones.

Sin embargo, antes de continuar profundizando en esto, es menester hacer una diferenciación entre la cobertura de los reportes que emiten las organización y los que emiten los gobiernos. Por una parte, los reportes que exponen las organizaciones abarcan desde indicadores de sostenibilidad organizativa hasta equidad de género y derechos humanos. Mientras que los informes que realizan los gobiernos abarcan medidas de progreso más allá del producto interno bruto (PIB), como los indicadores del estado del medio ambiente $\mathrm{y}$ cuadros de mando sobre la protección de los derechos humanos, entre otros (Tello, Hazelton, \& Cummings, 2016).

En el ámbito organizacional, Tello, Villiers et. al (2014) indicaron que la orientación de los informes debe ser predominantemente financiera. A diferencia de lo ejecutado por algunas entidades, quienes utilizaron partes de sus informes anuales para revelar información seleccionada sobre sus impactos sociales y ambientales y sus políticas para gestionar las interacciones entre la organización, la sociedad en la que operaba y el entorno natural. En su investigación, Tello et al. (2014) mencionan que estas divulgaciones sociales y ambientales dentro de los informes anuales, parecen haber sido motivadas en gran medida por el deseo de la organización o de la gerencia de cumplir con los requisitos de información percibidos por las partes interesadas que tenían el mayor poder económico en relación con una organización.
A pesar de ello, a medida que la información social y ambiental se fue extendiendo, las organizaciones comenzaron a utilizar otros medios además del informe anual para divulgar gran parte de esta información. Para muchas de estas organizaciones, el informe anual se centró en la comunicación de información de importancia fundamental a sus grupos de interés financieros.

Por otro lado, Bernard et al. (2018) enuncian que la influencia de los estados financieros en las decisiones económicas en general depende mucho de los encargados, ya que estos tienen la responsabilidad de establecer normas financieras que crean un mundo en el que la información de los datos y las decisiones están cada vez más entrelazadas, sin excluir la aplicación de los principios contables. Esto coincide con lo expuesto por Anil \& Stittle (2018) y Ghosh \& Wu (2012), quienes indican que el impacto de los estados financieros basados en los principios contables en cuanto a la mejora de la transparencia, la rendición de cuentas, la efectividad de las operaciones y las organizaciones, se traduce en el refuerzo de su control sobre los resultados, la financiación y los procesos presupuestarios.

Como se mencionó previamente, los encargados de enlazar las decisiones con la información financiera son, generalmente, los gerentes de finanzas. No obstante, al tratarse de decisiones que impactan en todas las áreas de la organización, estos no deben ser los únicos involucrados, sino también los empleados, desde el director financiero hasta los empleados de cuentas por pagar (Venkatesh \& Blaskovich, 2012). Por tanto, la participación diversificada (indistintamente del nivel) también constituye un factor influyente dentro de la relación "toma de decisiones - estados financieros".

En otras palabras, a pesar de que los gerentes son los responsables de generar informes financieros internos y externos (presupuestos, informes de desempeño, declaraciones de impuestos, etc.), estos deben basarse también en la información proporcionada por los subordinados, quienes tienen una visión más clara 
de la realidad de las entradas y salidas de dinero (Jollineau, Vance, \& Webb, 2012).

Cabe mencionar que los tomadores de decisiones deben estar intrínsecamente relacionados con el conocimiento de costos y el desempeño de los juicios basados en costos. En ese sentido, una contribución del estudio realizado por Demmer \& Shields (2001) demuestra que cuando los gerentes utilizan información de costos basada en el volumen sin el conocimiento de la metodología ABC o el de la actividad, esto afecta negativamente el desempeño de la evaluación basada en el costo. Por el contrario, mientras se tenga mayor conocimiento de $\mathrm{ABC}$ y de la actividad, aumentan el desempeño de la evaluación basada en el costo. Por aquello, se justifica la relación intrínseca que deben tener los tomadores de decisiones respecto a los costos, a fin de generar valor en la organización.

Por otro lado, es beneficioso considerar también la información financiera en la toma de decisiones respecto a préstamos, ya que es, precisamente, la información financiera la que permite conocer la capacidad o el estado de la empresa para asumir deuda. Dentro de los informes financieros que los inversionistas valoran mucho se encuentran: el estado de ganancias y pérdidas, el balance general, las notas a los estados financieros y el estado de flujo de caja (Schneider, 2018). Por ejemplo, en el caso de la selección de acciones, el análisis o examinación de estados financieros se considera una estrategia efectiva (Fiechter \& Novotny-farkas, 2017).

Lo mencionado anteriormente se sustenta con la existencia de pronósticos y ajustes estadísticos para la interrelación de los estados financieros y la toma de decisiones. De hecho, para Demmer et al. (2019), los analistas otorgan más credibilidad a las previsiones basadas en los estados financieros después de la adopción obligatoria de las NIIF en países con mejoras simultáneas en la aplicación de la información financiera $y$, en particular, si las previsiones se realizan en condiciones favorables. Los investigadores también explican que los pronósticos basados en estados financieros proporcionan información incremental para explicar las utilidades por acción del año siguiente.

\section{CONCLUSIONES}

Del presente estudio se destaca que los Estados Financieros per se constituyen indicadores de bienestar en las organizaciones, debido a que pueden plasman el estado económico de la entidad, así como su capacidad para generar valor y su grado de relación o cumplimiento con las estrategias empresariales y el modelo de negocio. Sin excluir el hecho de que existen ratios como la productividad $y$ responsabilidad social, que son útiles en la toma de decisiones y además proveen una presentación menos compleja para entender el funcionamiento de la actividad económica que se ejecuta en la organización.

Los beneficios de considerar la información financiera en la toma de decisiones no se limitan a tratar de alinear las estrategias empresariales con los resultados económicos futuros, sino también a mantener y mejorar la atracción de los inversionistas respecto a la organización. Como se mencionó previamente, los inversionistas buscan realizar un análisis en función de la rentabilidad de la empresa, para decidir si invertir o no en esta. Incluso cuando no se trate una inversión, sino de adquirir un préstamo, la información financiera es esencial.

Por ello, los tomadores de decisiones poseen una gran responsabilidad y eso conlleva a que estos se encuentren constantemente capacitados, no solo en cuestión de información financiera, sino también en cuestión de costos y la forma en que estos se generan. En ese sentido, la toma de decisiones no debe limitarse o asignarse únicamente al personal de nivel alto, sino también al personal de nivel medio y bajo. Esto se debe a que ellos permanecen al tanto del origen de las entradas y salidas, es decir, las operaciones que genera la empresa.

En resumen, el presente estudio afianza los conocimientos sobre la relación de los estados financieros y la toma de decisiones, resaltando que existen impactos positivos que no se limitan a la alianza entre las estrategias empresariales y 
los resultados, sino que van mucho más allá, como la diversificación en la participación de la toma de decisiones. Además, la credibilidad que obtendría la empresa frente a los inversionistas o al momento de acceder a un préstamo. De manera que, considerar toda la información financiera disponible en el proceso de toma de decisiones (de cualquier índole), genera un alto valor para la empresa y no debe ser más un tema de cuestionamiento, sino de práctica.

\section{REFERENCIAS}

Anil, N., \& Stittle, J. (2018). The role of accounting in transforming public tertiary institutions in New Zeland. Accounting, Auditing \& Accountability Journal, 31(2), 503-530.

doi:http://dx.doi.org.ezproxybib.pucp.edu .pe:2048/10.1108/AAAJ-09-2016-2722

Bernard, G., Dupuy, P., Baker, C., \& Blum, V. (2018). The impact of accounting standards on hedging decisions. Accounting \& Accountability Journal, 31(1), 193-213.

doi:http://dx.doi.org.ezproxybib.pucp.edu .pe:2048/10.1108/AAAJ-03-2016-2448

Boiral, O. (2013). Sustainability reports as simulacra? A counter-account of A and A+ GRI reports. Accounting, Auditing \& Accountability Journal, 26(7), 10361071.

doi:http://dx.doi.org.ezproxybib.pucp.edu .pe:2048/10.1108/AAAJ-04-2012-00998

Cheng, Q., Young, J., \& Yang, H. (2018).

Financial reporting changes and the internal information environment: Evidence from SFAS 142. Review of Accounting Studies, 23(1), 347-383. doi:http://dx.doi.org.ezproxybib.pucp.edu .pe:2048/10.1007/s11142-017-9437-8

Collins, D., Román, F., \& Chan, H. (2011). An empirical investigation of the relationship between profitability persistence and firms' choice of business model:
Evidence from the U.S. airline industry. Journal of Management Accounting Research, 23, 37-70. Obtenido de https://search-proquestcom.ezproxybib.pucp.edu.pe/docview/91 2867367 ?accountid=28391

Dearman, D., \& Shields, M. (2001). Cost knowledge and cost-based judgement perfomance. Journal of Management Accounting Research, 13, 1-18. Obtenido de https://search-proquestcom.ezproxybib.pucp.edu.pe/docview/21 0177079 ? accountid $=28391$

Demmer, M., Pronobis, P., \& Teri, L. (2019). Mandatory IFRS adoption and analyst characteristics. Review of Accounting Studies.

doi:http://dx.doi.org.ezproxybib.pucp.edu .pe:2048/10.1007/s11142-019-9481-7

Fiechter, P., \& Novotny-farkas, Z. (2017). The impact of the institutional environment on the value relevance of fair values. Review of Accounting Studies, 22(1), 392-429.

doi:http://dx.doi.org.ezproxybib.pucp.edu .pe:2048/10.1007/s11142-016-9378-7

Ghosh, D., \& Wu, A. (2012). The effect of positive and negative financial and nonfinancial performance measures on analyst's recommendations. Behavioral Research in Accounting, 24(2), 47-64. Obtenido de https://search-proquestcom.ezproxybib.pucp.edu.pe/docview/11 51907637 ?accountid $=28391$

Jollineau, J., Vance, T., \& Webb, A. (2012). Subordinates as the first line of defense against biased financial reporting. Journal of Management Accounting Research, 24, 1-24. Obtenido de https://search-proquestcom.ezproxybib.pucp.edu.pe/docview/12 47631983? accountid=28391 
Schneider, A. (2018). Studies on the impact of accounting information and assurance on commercial lending judgments. Journal of Accounting Literature, 41, 63-74. doi:http://dx.doi.org.ezproxybib.pucp.edu .pe:2048/10.1016/j.acclit.2018.03.005

Tello, E., Hazelton, J., \& Cummings, L. (2016). Potential user's perceptions of general purpose water accounting reports. Accounting, Auditing \& Accountability Journal, 20(1), 80-110. doi:http://dx.doi.org.ezproxybib.pucp.edu .pe:2048/10.1108/AAAJ-12-2013-1552

Venkatesh, R., \& Blaskovich, J. (2012). The mediating effect of psychological capital on the budget participation-job performance relationship. Journal of Management Accounting Research, 24, 159-175. Obtenido de https://searchproquestcom.ezproxybib.pucp.edu.pe/docview/12 47638908 ? accountid $=28391$

Villiers, C., Rinaldi, L., \& Unerman, J. (2014). Integrated reporting: Insights, gaps and an agenda for future research. Accounting, Auditing \& Accountability Journal, 27(7), 1042-1067. doi:http://dx.doi.org.ezproxybib.pucp.edu .pe:2048/10.1108/AAAJ-06-2014-1736 


\section{APÉNDICES}

TABLA 1

Resumen de revisión de literatura

\begin{tabular}{llcl}
\hline $\mathrm{N}^{\circ}$ & \multicolumn{1}{c}{ Autor } & Año & \multicolumn{1}{c}{ Título } \\
\hline 1 & $\begin{array}{l}\text { Bernard, G., Dupuy, } \\
\text { P., Baker, C. R., \& } \\
\text { Blum, V. }\end{array}$ & 2018 & $\begin{array}{l}\text { The impact of } \\
\text { accounting standards } \\
\text { on hedging decisions }\end{array}$ \\
& & & $\begin{array}{l}\text { Sustainability reports } \\
\text { as simulacra? A } \\
\text { counter-account of A } \\
\text { and A+ GRI reports. }\end{array}$
\end{tabular}

3 Tello, E., Hazelton, J., \& Cummings, L

de Villiers, C.,

4 Rinaldi, L., \&

Unerman, J.
Potential users' perceptions of general purpose water accounting reports

Potential users' perceptions of general purpose water accounting reports

The role of accounting in transforming public tertiary institutions in new zealand

The effect of positive and negative financial and nonfinancial performance measures on analysts' recommendations.

An empirical investigation of the relationship between profitability persistence and firms' choice of business model: Evidence from the U.S. airline industry.
Accounting,

Auditing \&

Accountability

Accounting, Auditing \& Accountability

Accounting, Auditing \& Accountability

Accounting, Auditing \& Accountability

Behavioral Research in Accounting

Journal of Management Accounting Research
El propósito de este documento es estudiar los efectos de las normas de contabilidad financiera en las decisiones económicas de los gerentes.

El propósito de este documento es examinar hasta qué punto los informes de sostenibilidad pueden ser utilizado para problemas reales de desarrollo sostenible y proyectar una visión idealizada de la situación de las empresas En particular, el documento examina en qué medida los usuarios consideran que los informes del GPWA son útiles y facilitan la rendición de cuentas por parte de los gestores del agua. El propósito de este documento es sintetizar las ideas de la investigación contable y de rendición de cuentas y propone una agenda completa para la investigación futura en este campo

El propósito de este trabajo es identificar y evaluar el papel y la influencia de la disciplina contable a través de su asociación con las múltiples lógicas de las reformas gubernamentales para transformar el sector público de la educación terciaria en Nueva Zelanda.

Esta investigación examina experimentalmente los resultados favorables y desfavorables de las medidas de rendimiento financiero y no financiero de una empresa en base a la recomendación de los analistas financieros de desinvertir $o$ invertir en una empresa

Este documento examina la influencia del modelo de negocio en la rentabilidad. 
8

Venkatesh, R., \&

Blaskovich, J. (2012).

Jollineau, S. J., Vance,

9 T. W., \& Webb, A. (2012)..

10

Dearman, D. T., \&

Shields, M. D.

2001

Cost knowledge and cost-based judgment performance

Subordinates as the first line of defense against biased financial reporting

The mediating effect of psychological capital on the budget participation-job performance relationship.

Studies on the impact of accounting

11 Schneider, A

2018 information and assurance on commercial lending judgments.

Fundamental analysis of banks: The use of

12 Mohanram, P., Saiy, S., \& Vyas, D.

2018 financial statement information to screen winners from losers.

Mandatory IFRS adoption and analyst

Demmer, M.,

13 Pronobis, P., \& Teri, L. Y.

forecast accuracy: The

role of financial

statement-based

forecasts and analyst

characteristics.

The impact of the

institutional

2017

Fiechter, P., \&

Novotny-farkas, Z. environment on the

value relevance of fair values.
Journal of Management Accounting Research

Journal of Management Accounting Research

Journal of Management Accounting Research

Journal of

Accounting

Literature

Review of

Accounting

Studies

Review of

Accounting

Studies

Review of

Accounting

Studies
Este estudio se basa en la literatura sobre psicología positiva y comportamiento organizacional para examinar el papel del capital psicológico de un individuo (PsyCap) en la relación entre participación presupuestaria $\mathrm{y}$ desempeño en el trabajo.

Los gerentes que generan informes financieros a menudo dependen de subordinados que poseen información privada para proporcionar insumos. Cuando los gerentes tienen incentivos para manipular los informes, pueden solicitar aportaciones sesgadas de los subordinados.

Este documento presenta evidencia sobre cómo el conocimiento de los costos de los gerentes afecta el desempeño de sus juicios basados en costos cuando se utiliza un sistema de contabilidad de costos basado en el volumen y los productos tienen un consumo diverso de recursos.

En este artículo se revisan estudios que han examinado cómo la información contable afecta a los juicios sobre préstamos comerciales.

Este estudio investiga la eficacia de un enfoque basado en un análisis fundamental para evaluar las acciones de los bancos de EE.UU.

Este estudio examina si la mejora en la precisión de las previsiones de los analistas en torno a la adopción obligatoria de las NIIF está asociada a la mejora de la precisión de las previsiones basadas en los estados financieros. En estudio explica que las diferencias institucionales entre países (por ejemplo, el entorno de información o la sofisticación del mercado) afectan a la capacidad de los inversores para procesar y retener información sobre el valor razonable en su valoración.

Fuente: Los autores. 